\title{
Epidendrum secundum Jacq. e E. denticulatum Barb. Rodr. (Orchidaceae): caracteres úteis para a sua delimitação
}

\author{
Fábio Pinheiro ${ }^{1,3}$ e Fábio de Barros ${ }^{2}$
}

Recebido: 28.06.2007; aceito: 22.11.2007

\begin{abstract}
Epidendrum secundum Jacq. and E. denticulatum Barb. Rodr. (Orchidaceae): useful characters for their recognition). Epidendrum L. is the largest genus of Orchidaceae in the Neotropical region, with about 1,125 species. The extensive variation that is present in several species turns the delimitation of the genus and of some species a quite controversial theme. Epidendrum secundum and E. denticulatum belong to section Amphyglottium, which embraces some of the most variable species within the genus. Apart the inclusion of Epidendrum secundum and E. denticulatum in different subsections, due to the contrasting morphology of the lip callus, these species have several similarities, in the habit, morphology and flower color. As in many herbarium specimens the visualization of the lip callus is difficult; the overall similarity between the species has caused several misidentifications in herbaria as well as in living plants. The aim of this work is to select new characters set to be used for a more accurate identification of the herbarium registers of E. secundum and $E$. denticulatum. Thirty individuals of E. secundum and 26 of $E$. denticulatum maintained under cultivation in the orchids collection of the Institute of Botany of São Paulo were sampled, and 20 quantitative characters were measured from them. Methods of multivariate analysis (PCA, UPGMA, DA and Box-Plot) and Student's t Test, corrected by the Bonferroni criterion, were employed. Six floral characters have a significant difference between E. secundum and E. denticulatum, showing that morphometric techniques can be useful for the delimitation of variable species. An identification key is presented for E. secundum and E. denticulatum.
\end{abstract}

Key words: Morphological variation, morphometry, multivariate analysis, taxonomy

RESUMO - (Epidendrum secundum Jacq. e E. denticulatum Barb. Rodr. (Orchidaceae): caracteres úteis para sua delimitação). Epidendrum L. é o maior gênero de Orchidaceae na região Neotropical, com cerca de 1.125 espécies. A grande variação que diversas espécies apresentam torna a delimitação do gênero e das próprias espécies um tema bastante controverso. Epidendrum secundum e E. denticulatum são espécies que pertencem à Seção Amphyglottium, a qual inclui algumas das espécies mais variáveis do gênero. Apesar de pertencerem a subseções distintas, devido à morfologia diversa do calo do labelo, são espécies que apresentam muitas semelhanças, no porte geral e morfologia das plantas e na coloração das flores. Como em muitos materiais de herbário a visualização do calo do labelo é difícil, esta semelhança entre as espécies tem gerado diversas identificações trocadas, tanto em herbários quanto em plantas vivas. O objetivo deste trabalho é selecionar um conjunto de caracteres que possa ser utilizado para uma identificação mais precisa dos registros de E. secundum e E. denticulatum. Foram amostrados 30 indivíduos de E. secundum e 26 de E. denticulatum mantidos em cultivo na Seção de Orquidário do Instituto de Botânica de São Paulo, e destes indivíduos foram medidos 20 caracteres quantitativos. Foram empregados métodos de análise multivariada (PCA, UPGMA, DA e Box-Plot) e o Teste t de Student, corrigido pelo critério de Bonferroni. Foram encontrados seis caracteres florais que possuem uma diferença significativa entre E. secundum e E. denticulatum, revelando que técnicas de morfometria podem ser bastante úteis para a delimitação de espécies amplamente variáveis, de difícil circunscrição. É fornecida uma chave para separação entre E. secundum e E. denticulatum.

Palavras-chave: análise multivariada, morfometria, taxonomia, variação morfológica

\section{Introdução}

O gênero Epidendrum L. é um dos maiores da família Orchidaceae, com cerca de 1.125 espécies (Chase et al. 2003). O gênero é bastante variável morfologicamente e mal compreendido taxonomicamente, já que sua delimitação genérica e a posição sistemática de muitas de suas espécies

1. Universidade de São Paulo, Instituto de Biociências, Departamento de Botânica, Cidade Universitária, Rua do Matão 277, 05508-900 São Paulo, SP, Brasil

2. Instituto de Botânica, Caixa Postal 3005, 01061-970 São Paulo, SP, Brasil

3. Autor para correspondência: biopinheiro@yahoo.com.br 
têm sido alvos de controvérsia (Dressler 1967, 1984, Brieger 1976-1977, Hágsater 1993, Withner \& Harding 2004).

Dentro do gênero, um grupo que abriga algumas das espécies mais variáveis morfologicamente foi delimitado primeiramente por Lindley (1852-1859), constituindo o subgênero Amphiglottium, composto por diversas seções, entre as quais a seção Schistochila. As espécies desta seção foram distribuídas em duas subseções segundo a morfologia do calo do labelo: subseção Carinata, com um calo central em forma de quilha e projetado longitudinalmente sobre o lobo central (figura 1a) e subseção Tuberculata, com calo carnoso, variadamente recortado, geralmente limitado ao centro do disco (figura 1b). Cogniaux (1898-1902), Pabst \& Dungs (1975) e Brieger (19761977) seguiram, em grande parte, a subdivisão proposta por Lindley (1852-1859), transformando o subgênero Amphiglottium em seção Amphiglottium, e distribuindo as espécies nas subseções Carinata e Tuberculata, utilizando os mesmos critérios morfológicos de Lindley (1852-1859).

Apesar de pertencerem a subseções distintas, E. secundum Jacq. (subseção Tuberculata) e E. denticulatum Barb. Rodr. (subseção Carinata) possuem um hábito bastante similar (caule cilíndrico com folhas dísticas, coriáceas, inflorescência do mesmo comprimento ou mais longa que o caule, com flores geralmente dispostas em corimbo), flores bastante similares, de mesmo tamanho, e róseas a lilases. A diferença mais marcante está no calo do labelo, caráter que permite o posicionamento destas espécies em subseções distintas: E. secundum possui um calo carnoso, recortado de diversas formas (figura 1a, f, g, h), enquanto $E$. denticulatum possui dois calos carnosos arredondados na base e um calo disposto longitudinalmente, em forma de quilha (figura $1 \mathrm{~b}, \mathrm{c}$, $\mathrm{d}, \mathrm{e})$.
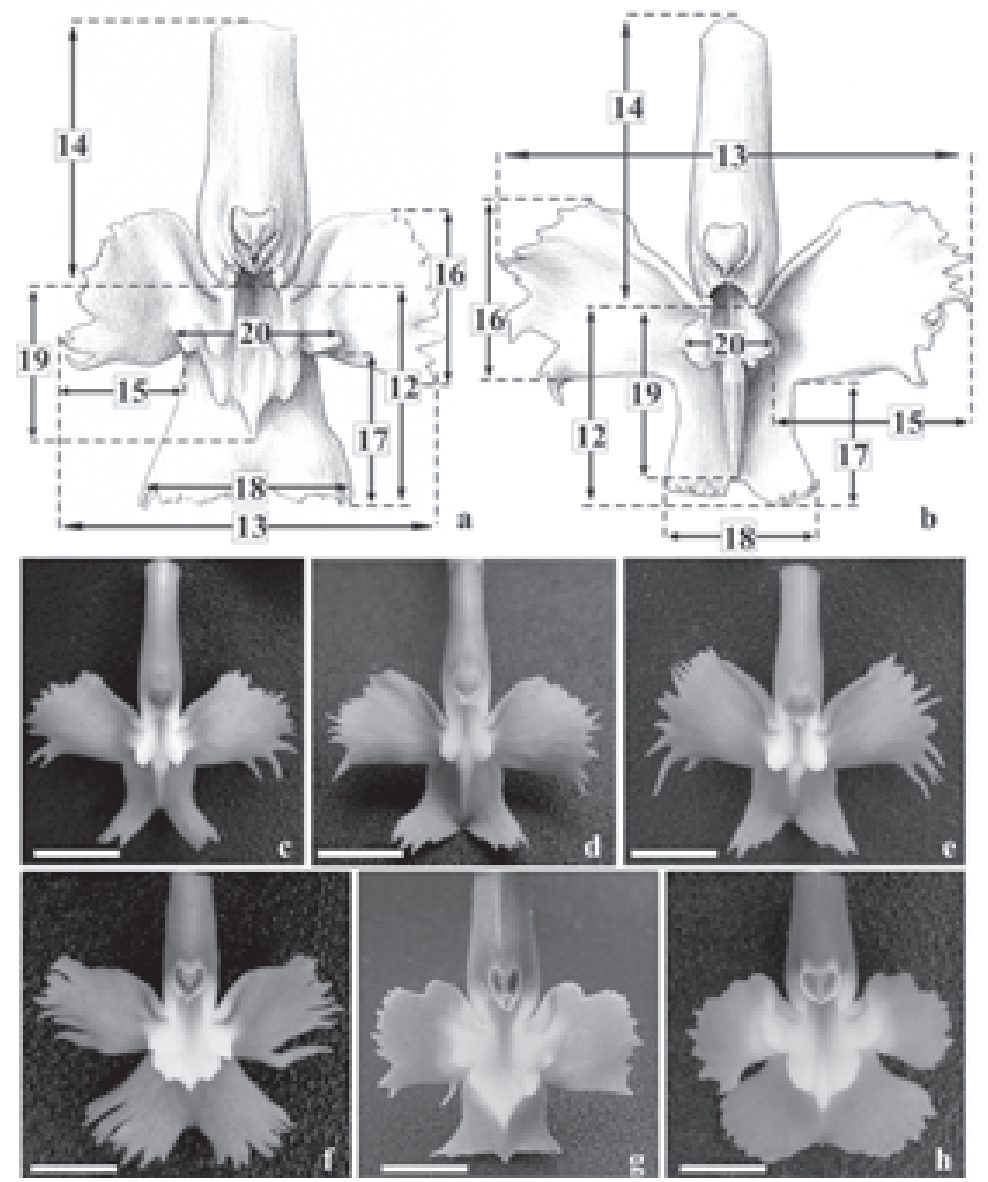

Figura 1. Variação morfológica da coluna e labelo de flores de E. denticulatum e E. secundum. a-b. Desenho de coluna e labelo de E. secundum e E. denticulatum, respectivamente, mostrando os caracteres medidos. Os números indicam os caracteres listados na tabela 2. c-e. Coluna e labelo de indivíduos de E. denticulatum mostrando a variação morfológica existente na espécie (indivíduos procedentes, respectivamente, de São Gonçalo do Rio Abaixo - MG, Araruama - RJ e Bertioga - SP). f-h. Coluna e labelo de indivíduos de E. secundum mostrando a variação morfológica existente na espécie (indivíduos procedentes, respectivamente, de Diamantina - MG, Nova Friburgo - RJ e Alto Paraíso - GO). Barra de escala = $4 \mathrm{~mm}$. 
Um levantamento realizado em herbários com grandes coleções de Orchidaceae (SP, SPF, HB, RB, $\mathrm{R})$ revelou que diversos registros de E. secundum estão identificados como E. denticulatum e viceversa. Isto ocorre devido à grande dificuldade em se detectar as diferenças no calo do labelo de flores de espécimes herborizados, e uma identificação precisa só é possível com a fervura e dissecação das flores. Tal confusão também tem levado à identificação trocada em plantas vivas em coleções e exposições de orquídeas.

A identificação de espécies próximas, que possuem poucos caracteres qualitativos diagnósticos, pode ser aperfeiçoada com o emprego da morfometria e de técnicas de análise multivariada, que permitem a avaliação de diversos caracteres quantitativos simultaneamente. Este enfoque é bastante comum em grupos de espécies de Orchidaceae (Borba et al. 2002, Goldman et al. 2004), trazendo, em alguns casos, subsídios para a descrição de novas espécies (Palestina \& Sosa 2002), constituindo uma metodologia de trabalho robusta, que permite a delimitação mais objetiva das espécies (Jensen 2003, Hendersson 2005).

O objetivo deste trabalho é identificar caracteres quantitativos que possam ser utilizados para diferenciar, com maior precisão, indivíduos de E. secundum e de E. denticulatum.

\section{Material e métodos}

Foram utilizados 30 indivíduos de E. secundum e 26 indivíduos de E. denticulatum, mantidos sob cultivo na Seção de Orquidário do Instituto de Botânica (São Paulo, SP, Brasil). As medidas foram tomadas a partir de material fresco porque, nessa condição, os caracteres qualitativos são facilmente observados, e a tomada das medidas é facilitada porque pode ser feita nas flores intactas. Um teste piloto foi realizado para comparar os resultados obtidos de medidas tomadas a partir de flores frescas e a partir de flores herborizadas e dissecadas, todas de um mesmo indivíduo, e os resultados não apresentaram diferença significativa $(\mathrm{P}<0,0005)$.

O critério para escolha dos indivíduos foi o de incluir na análise espécimes que: (a) cobrissem a variação morfológica das espécies, avaliada através de consulta de materiais dos herbários SP, SPF, UEC, $\mathrm{R}, \mathrm{RB}, \mathrm{BR}$, e MBM; e (b) cobrissem também os diferentes tipos de hábitat nos quais as espécies ocorrem predominantemente - restinga e cerrado para
E. denticulatum, Mata Atlântica de Altitude e Campo Rupestre para E. secundum -, amostrando populações destes ambientes. Materiais representativos foram prensados e depositados no Herbário SP (tabela 1). Vinte caracteres quantitativos contínuos foram medidos, sendo quatro vegetativos e 16 florais (tabela 2), utilizando paquímetro digital. Os caracteres vegetativos foram medidos diretamente na planta em cultivo, enquanto os caracteres florais foram medidos em flores conservadas em álcool $70 \%$.

A análise de componentes principais (Principal Component Analysis - PCA) foi empregada para representar graficamente a variação morfológica entre ambas as espécies, numa tentativa de detectar padrões de variação contínua e saber quais caracteres contribuem para o padrão observado. O método de agrupamento UPGMA (Unweighted Pair Group Method with Arithmetic Average) foi empregado para detectar descontinuidades morfológicas entre as espécies, inclusive entre as populações dentro de cada espécie, utilizando distância euclidiana simples. $\mathrm{O}$ Teste $\mathrm{t}$ de Student foi empregado para medir a significância das diferenças encontradas entre os caracteres das duas espécies, tendo os valores das probabilidades corrigidos pelo critério de Bonferroni. AAnálise Discriminante (DA) foi utilizada para testar a significância das diferenças encontradas, considerando as interações e correlações entre todos os caracteres. Caracteres altamente correlacionados foram retirados da análise pelo método de stepwise, para que a classificação dos indivíduos fosse mais robusta. As análises foram realizadas com os

Tabela 1. Procedência dos 56 indivíduos utilizados nas análises. Espécimes representativos foram depositados no herbário SP.

\begin{tabular}{|c|c|c|}
\hline Espécie/Procedência & $\mathrm{N}$ & Voucher \\
\hline \multicolumn{3}{|l|}{ E. denticulatum } \\
\hline São Gonçalo do Rio Abaixo - MG & 5 & Pinheiro 441 \\
\hline Araruama - RJ & 5 & Pinheiro 442 \\
\hline Bertioga - SP & 5 & Pinheiro 443 \\
\hline Itirapina - SP & 5 & Pinheiro 444 \\
\hline Mogi-Guaçú - SP & 6 & Pinheiro 445 \\
\hline \multicolumn{3}{|l|}{ E. secundum } \\
\hline Alto Paraíso - GO & 5 & Pinheiro 440 \\
\hline Diamantina - MG & 5 & $\begin{array}{c}\text { Brólio \& Silva s.n. } \\
\quad \text { (SP365931) }\end{array}$ \\
\hline Nova Friburgo - RJ & 5 & Pinheiro et al. \\
\hline Atibaia - SP & 5 & Pinheiro \& Inês 202 \\
\hline Cananéia - SP & 5 & Pinheiro 446 \\
\hline São Bernardo do Campo - SP & 5 & Pinheiro \& Inês 226 \\
\hline
\end{tabular}


Tabela 2. Caracteres utilizados nas análises, suas respectivas siglas, médias e desvios-padrão, valores das probabilidades medidas pelo Teste $\mathrm{t}(\mathrm{T})$ ajustadas pelo método de Bonferroni (B), e os três primeiros autovalores na análise de PCA (PC1, PC2 e PC3, respectivamente).

\begin{tabular}{|c|c|c|c|c|c|c|c|}
\hline \multirow[t]{2}{*}{ Caráter } & \multirow[t]{2}{*}{ E. secundum } & \multirow[t]{2}{*}{ E. denticulatum } & \multicolumn{2}{|c|}{ Significância ${ }^{1}$} & \multirow[t]{2}{*}{ PC1 } & \multirow[t]{2}{*}{ PC2 } & \multirow[t]{2}{*}{ PC3 } \\
\hline & & & $\mathrm{T}$ & $\mathrm{B}$ & & & \\
\hline 1. Comprimento da folha (C-FOL) & $86,700 \pm 13,983$ & $78,269 \pm 11,188$ & $*$ & n.s. & $-0,003$ & 0,193 & 0,401 \\
\hline 2. Largura da folha (L-FOL) & $23,767 \pm 4,636$ & $24,308 \pm 3,845$ & n.s. & n.s. & 0,096 & $-0,027$ & 0,423 \\
\hline 3. Comprimento do caule (C-CAU) & $503,667 \pm 174,385$ & $471,154 \pm 154,824$ & n.s. & n.s. & $-0,054$ & 0,138 & 0,407 \\
\hline 4. Comprimento da inflorescência (C-INF) & $396,333 \pm 170,607$ & $465,000 \pm 194,592$ & n.s. & n.s. & 0,041 & $-0,062$ & 0,456 \\
\hline 5. Comprimento do pedicelo (C-PED) & $23,727 \pm 4,193$ & $23,378 \pm 4,580$ & n.s. & n.s. & 0,216 & 0,204 & 0,133 \\
\hline 6. Comprimento da sépala dorsal (C-SED) & $9,896 \pm$ & $10,650 \pm$ & $* *$ & n.s. & 0,341 & $-0,030$ & $-0,148$ \\
\hline 7. Largura da sépala dorsal (L-SED) & $3,919 \pm$ & $4,311 \pm$ & $* *$ & $*$ & 0,269 & $-0,035$ & 0,188 \\
\hline 8. Comprimento da sépala lateral (C-SEL) & $10,452 \pm$ & $11,074 \pm$ & $*$ & n.s. & 0,336 & 0,018 & $-0,165$ \\
\hline 9. Largura da sépala lateral (L-SEL) & $4,523 \pm$ & $4,704 \pm$ & n.s. & n.s. & 0,236 & 0,070 & 0,198 \\
\hline 10. Comprimento da pétala (C-PET) & $10,181 \pm$ & $10,900 \pm$ & $*$ & n.s. & 0,344 & $-0,008$ & $-0,142$ \\
\hline 11. Largura da pétala (L-PET) & $3,670 \pm$ & $3,550 \pm$ & n.s. & n.s. & 0,193 & 0,234 & 0,146 \\
\hline 12. Comprimento do labelo (C-LAB) & $5,288 \pm$ & $5,192 \pm$ & n.s. & n.s. & 0,267 & 0,227 & $-0,205$ \\
\hline 13. Largura do labelo (L-LAB) & $10,032 \pm$ & $12,575 \pm$ & $* * *$ & $* * *$ & 0,291 & $-0,229$ & 0,077 \\
\hline 14. Comprimento da coluna (C-CO) & $5,431 \pm$ & $7,562 \pm$ & $* * *$ & $* * *$ & 0,245 & $-0,319$ & 0,062 \\
\hline $\begin{array}{l}\text { 15. Comprimento do lobo lateral do labelo } \\
\text { (C-LLL) }\end{array}$ & $3,200 \pm$ & $5,323 \pm$ & $* * *$ & $* * *$ & 0,227 & $-0,361$ & 0,049 \\
\hline 16. Largura do lobo lateral do labelo (L-LLL) & $4,504 \pm$ & $5,261 \pm$ & $* *$ & n.s. & 0,258 & $-0,091$ & 0,105 \\
\hline $\begin{array}{l}\text { 17. Comprimento do lobo central do labelo } \\
\text { (C-LCL) }\end{array}$ & $3,427 \pm$ & $2,719 \pm$ & $* * *$ & $* * *$ & 0,080 & 0,398 & $-0,070$ \\
\hline 18. Largura do lobo central do labelo (L-LCL) & $6,893 \pm$ & $6,152 \pm$ & $* *$ & n.s. & 0,080 & 0,336 & 0,015 \\
\hline 19. Comprimento do calo do labelo (C-CAL) & $4,060 \pm$ & $4,068 \pm$ & n.s. & n.s. & 0,282 & 0,195 & $-0,185$ \\
\hline 20. Largura do calo do labelo (L-CAL) & $3,850 \pm$ & $2,415 \pm$ & $* * *$ & $* * *$ & $-0,002$ & 0,425 & 0,010 \\
\hline
\end{tabular}

${ }^{1}$ Níveis de significância: n.s. = não significativo para: $\mathrm{P} \geq 0,05 ; *=$ significativo para $\mathrm{P}<0,05 ; * *=$ significativo para $\mathrm{P}<0,01$; $* * *=$ significativo para $\mathrm{P}<0,001$.

programas Fitopac 1.6.4 (Shepherd 2006) e SYSTAT 10 (Wilkinson 2000).

\section{Resultados}

Os resultados são bastante semelhantes entre os métodos utilizados, indicando uma clara diferenciação morfológica entre E. secundum e E. denticulatum, e revelando que os caracteres quantitativos utilizados possuem grau de descontinuidade morfológica suficiente para que os táxons sejam reconhecidas como espécies distintas.

Os gráficos de PCA, construídos com os três primeiros eixos, reúnem cerca de $67 \%$ da variação do conjunto analisado e revelam uma tendência de separação entre indivíduos de E. secundum e E. denticulatum (figura 2). A separação entre indivíduos das duas espécies está melhor representada ao longo do eixo dois, no qual os caracteres comprimento do lobo lateral do labelo (C-LLL), comprimento da coluna (C-CO) e largura do labelo (L-LAB) possuem os maiores valores para indivíduos de $E$. denticulatum, enquanto que a largura do calo do labelo (L-CAL), comprimento e largura do lobo central do labelo (C-LCL e L-LCL) possuem as maiores dimensões para indivíduos de E. secundum (figura 2). A maior parte dos caracteres esta correlacionada ao eixo um, e provavelmente representam a variação de tamanho geral entre os indivíduos, e não diferenças relacionadas à forma. Não é possível reconhecer um padrão na variação representada pelo terceiro eixo (figura 2).

No dendrograma de agrupamento também é possível reconhecer uma clara diferenciação morfológica entre indivíduos de E. denticulatum, que formam um único agrupamento, e de E. secundum (figura 3), que está distribuído em grupos na parte mais externa do dendrograma. Apenas dois indivíduos, um de cada espécie, não se agruparam com indivíduos da mesma espécie. Não é possível observar grupos que reúnem indivíduos de uma mesma população (figura 3).

A maior parte dos caracteres utilizados exibe 

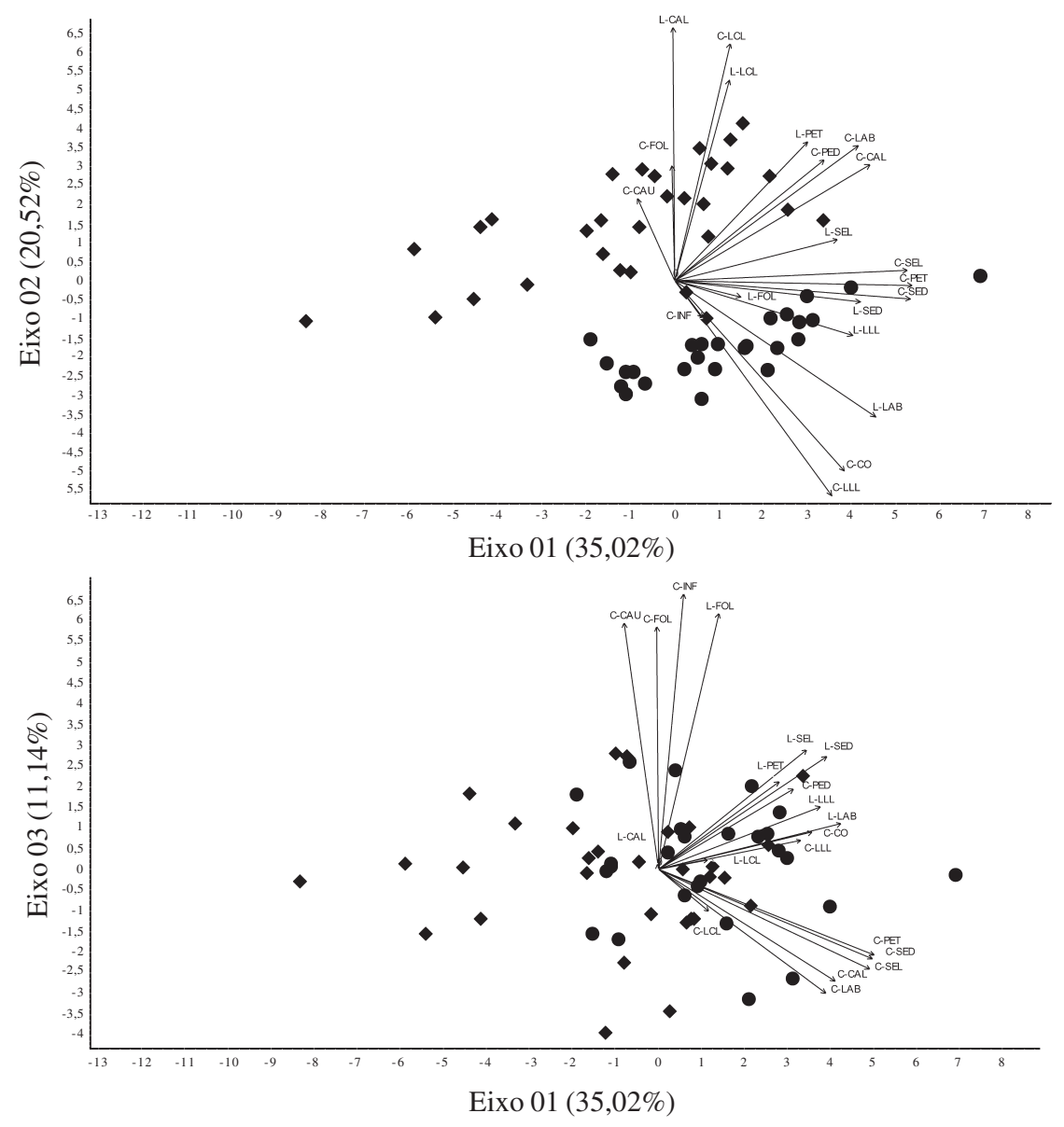

Figura 2. Gráfico de PCA de 26 indivíduos de E. denticulatum (•) e 30 indivíduos de E. secundum ( $\bullet$ ), baseado em 20 caracteres (ver tabela 2). Eixos 1, 2 e 3 concentram, respectivamente, 35,02\%, 20,52\% e 11,14\% da variância total da amostra. Valores esperados de variância segundo o modelo de Broken-stick são, respectivamente, 17,98\%, 12,98\% e 10,48\%.

sobreposição de tamanho, revelado pelos gráficos de box-plot (dados não apresentados), o que explica, parcialmente, porque as duas espécies são comumente confundidas. A largura do labelo (L-LAB), comprimento da coluna (C-CO), comprimento do lobo lateral do labelo (C-LLL), comprimento do lobo central do labelo (C-LCL) e largura do calo do labelo (LCAL) são os caracteres que exibem maior descontinuidade morfológica (figura 4).

$\mathrm{O}$ Teste $\mathrm{t}$ revelou que a maior parte dos caracteres não possui uma diferença significativa entre as duas espécies (tabela 2). A diferença encontrada entre E. secundum e E. denticulatum baseada nos caracteres C-FOL, C-SED, C-SEL, C-PET, L-LLL e L-LCL foi considerada significativa pelo Teste t, mas não após a realização da correção de Bonferroni, que apresentou resultados contrários (tabela 2). Os caracteres que mantiveram diferenças significativas após a correção de Bonferroni foram a largura da sépala dorsal (L-SED), a largura do labelo (L-LAB), o comprimento da coluna (C-CO), o comprimento do lobo lateral do labelo (C-LLL), o comprimento do lobo central do labelo (C-LCL) e a largura do calo (L-CAL).

A Análise Discriminante revelou que as diferenças encontradas entre as duas espécies possuem alto grau de significância (Wilk's lambda com $\mathrm{P}=0,0000)$. Na matriz de classificação por Jackknife, $98 \%$ dos indivíduos foram classificados como pertencentes às espécies previamente reconhecidas (tabela 3 ). Foram retirados da análise nove caracteres que possuíam alto grau de correlação (tabela 4). Apenas 11 caracteres foram utilizados para produzir a matriz de classificação por Jackknife da tabela 3. O comprimento do lobo lateral do labelo (C-LLL), o comprimento da coluna (C-CO), o comprimento da folha (C-FOL), o comprimento do caule (C-CAU) e o comprimento do lobo central do 
labelo (C-LCL) foram os cinco caracteres que exibiram menor correlação entre si e que, portanto, mostraram maior importância na análise.

\section{Discussão}

Apesar de E. denticulatum e E. secundum terem se revelado bastante semelhantes morfologicamente nas análises realizadas, foi possível detectar caracteres com significativa descontinuidade morfológica, que poderão ser utilizados para subsidiar a identificação de espécimes de ambas as espécies.

Muitos autores acreditam que os indivíduos classificados como E. secundum podem pertencer a diversas espécies, constituindo o "complexo E. secundum", um grupo informal constituído por

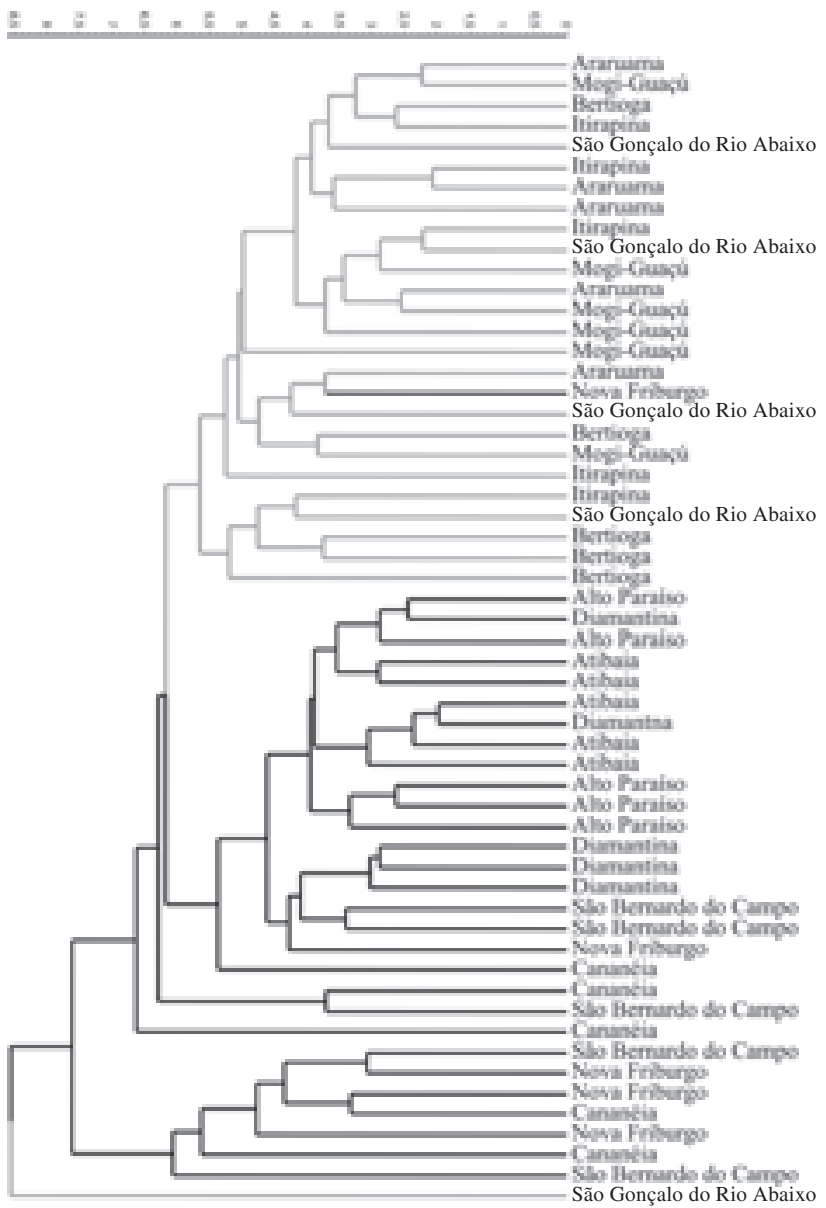

Figura 3. Dendrograma de UPGMA de 26 indivíduos de E. denticulatum (linhas cinza) e 30 indivíduos de E. secundum (linhas pretas), baseado em 20 caracteres (ver tabela 2), utilizando coeficiente de distância euclidiana simples. Indivíduos provenientes de uma mesma população ocupam diferentes agrupamentos. Correlação cofenética $=0,74$. diversos táxons com delimitação difícil e controversa (Pabst \& Dungs 1975, Brieger 1976-1977, Dunsterville 1979, Dressler 1989, Sastre 1990a, b). A grande variação morfológica presente em indivíduos de E. secundum, maior que aquela exibida por indivíduos de E. denticulatum, pode estar relacionada à presença de mais de uma espécie entre os indivíduos classificados, neste trabalho, como E. secundum. A utilização de morfometria, em conjunto com dados moleculares do tipo AFLP e microssatélites, capazes de discriminar grande quantidade de polimorfismos, podem ser bastante úteis neste complexo, auxiliando na identificação de linhagens e espécies distintas e contribuindo para um maior entendimento taxonômico do grupo.

Caracteres florais foram os mais importantes na delimitação das duas espécies, revelando descontinuidades morfológicas significativas (figura 4; tabelas 2, 4), sendo eles: largura da sépala dorsal (L-SED), largura do labelo (L-LAB), comprimento da coluna (C-CO), comprimento do lobo lateral do labelo (C-LLL), comprimento do lobo central do labelo (C-LCL) e largura do calo do labelo (L-CAL). Trabalhos semelhantes também indicaram que caracteres florais foram os mais importantes na delimitação de grupos de espécies afins (Borba et al. 2002, Palestina \& Sosa 2002). Os autores atribuem este fato a mecanismos de polinização diferenciados entre as espécies, com o aproveitamento de polinizadores distintos, porém em E. secundum e $E$. denticulatum, não existem evidências de que exista uma relação de especificidade com os respectivos polinizadores (Pansarin 2000). O tipo de hábitat em que as espécies ocorrem pode ser um dos fatores responsáveis pela diferenciação morfológica entre elas: $E$. denticulatum ocorre principalmente em dois tipos de vegetação, restinga e cerrado, enquanto $E$. secundum ocorre predominantemente em áreas montanhosas.

Apesar de E. secundum e E. denticulatum serem bastante semelhantes, a ponto de possuírem registros de herbário com identificação trocada, os dados morfométricos indicaram que ambas as espécies possuem uma diferenciação estatisticamente significativa, e que alguns caracteres podem ser utilizados para o reconhecimento de cada uma delas.

Uma chave de identificação para as espécies é apresentada a seguir, baseada nos caracteres que apresentaram diferença estatística significativa revelada pelo Teste $t$ corrigido pelo critério de 

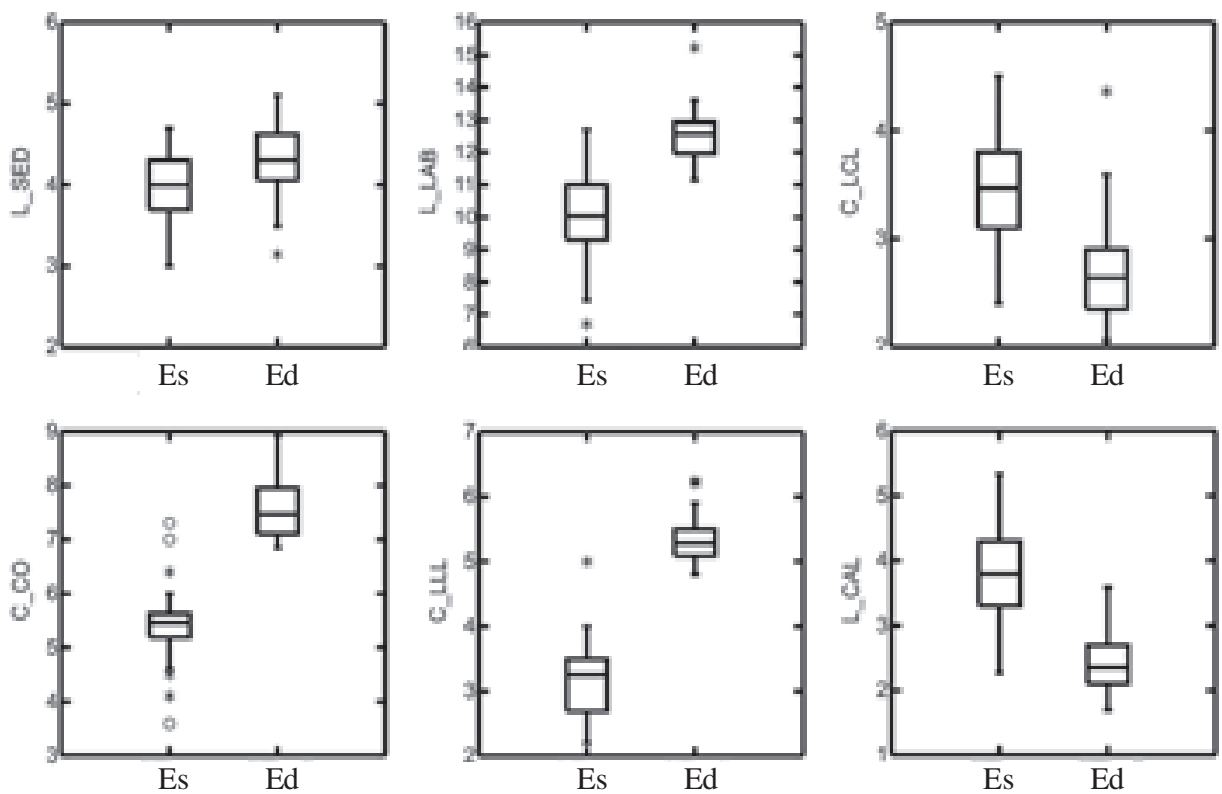

Figura 4. Gráficos box plot dos seis caracteres quantitativos medidos em indivíduos de E. denticulatum (Ed) e E. secundum (Es) que revelaram diferença significativa segundo o Teste t ajustado pelo método de Bonferroni (ver tabela 2). Retângulos definem os valores de 25 e 75 percentis; linhas horizontais mostram a mediana; linhas verticais definem os limites de 10 a 90 percentis; asteriscos e círculos indicam indivíduos com valores extremos. As siglas dos caracteres são apresentadas na tabela 2.

Tabela 3. Resultados da matriz de classificação gerada pela Análise Discriminante, utilizando o método de Jackknife Wilks' lambda com $\mathrm{P}=0,0000$.

\begin{tabular}{lccc}
\hline Grupos reconhecidos a priori & E. denticulatum & E. secundum & \% de agrupamentos corretos \\
\hline E. denticulatum & 26 & 0 & 100 \\
E. secundum & 1 & 29 & 97 \\
Total & 27 & 29 & 98 \\
\hline
\end{tabular}

Tabela 4. Valores de "F-to-remove" para os caracteres que permaneceram na análise discriminante, após seleção realizada pelo método de stepwise, por possuírem baixa correlação entre si, e valores de "F-to-enter" para os caracteres que foram retirados da análise por apresentarem alta correlação entre si.

\begin{tabular}{cccc}
\hline Caracteres que permaneceram na análise & "F-to-remove" & Caracteres que foram retirados da análise & "F-to-enter" \\
\hline C-FOL & 22,02 & L-FOL & 0,43 \\
C-CAU & 16,64 & C-PED & 0,00 \\
C-INF & 3,08 & C-SED & 0,57 \\
L-SED & 2,93 & C-SEL & L-SEL \\
C-PET & 4,61 & C-LAB & 0,14 \\
L-PET & 4,51 & L-LCL & 0,11 \\
L-LAB & 7,21 & C-CAL & 0,23 \\
C-CO & 25,97 & L-CAL & 1,24 \\
C-LLL & 66,34 & & 0,40 \\
L-LLL & 4,38 & & \\
C-LCL & 8,28 & \\
\hline
\end{tabular}




\section{Bonferroni (tabela 2):}

1. Largura da sépala dorsal $3,4-4,4 \mathrm{~mm}$; largura do labelo 8,6-11,4 mm; comprimento da coluna 4,7-6,2 mm; comprimento do lobo lateral do labelo 2,5-3,8 mm; comprimento do lobo central do labelo $3-4 \mathrm{~mm}$; largura do calo do labelo 3-4,6 mm; labelo com um calo carnoso, recortado de diver sas formas E. secundum

1. Largura da sépala dorsal $3,8-4,7 \mathrm{~mm}$; largura do labelo 11,7-13,4 mm; comprimento da coluna $7-8 \mathrm{~mm}$; comprimento do lobo lateral do labelo 4,9-5,7 mm; comprimento do lobo central do labelo 2,2-3,2 $\mathrm{mm}$; largura do calo do labelo 2-2,8 $\mathrm{mm}$; labelo com dois calos basais, arredondados, e um calo central, em forma de quilha, projetado longitudinalmente sobre o lobo central E. denticulatum

\section{Agradecimentos}

Aos funcionários de apoio da Seção de Orquidário do Estado / Instituto de Botânica. A Eduardo Leite Borba pela revisão deste artigo. A Rubens Custódio da Mota, Cláudio N. de Fraga, Melissa F. Bocayuva Cunha e Emerson R. Pansarin por fornecerem parte dos espécimes analisados. À FAPESP pela bolsa de mestrado recebida pelo primeiro autor (processo 03/03063-1) e ao CNPq pela bolsa de produtividade em pesquisa recebida pelo segundo autor (processo 303962/2004-6).

\section{Literatura citada}

Borba, E.L., Shepherd, G.J., van den Berg, C. \& Semir, J. 2002. Floral and vegetative morphometrics of five Pleurothallis (Orchidaceae) species: correlation with taxonomy, phylogeny, genetic variability and pollination systems. Annals of Botany 90: 219-230.

Brieger, F.G. 1976-1977. Gattungsreihe Epidendra. In: F.G. Brieger, R. Maatsch \& K. Senghas (eds.). Schlechter, Die Orchideen. Paul Parey, Berlin, v.3, pp. 509-549.

Chase, M.W., Cameron, K.M., Barrett, R.L. \& Freudenstein, J.V. 2003. DNA data and Orchidaceae systematics: a new phylogenetic classification. In: K.W. Dixon, S.P. Kell, R.L. Barrett \& P.J. Cribb (eds.). Orchid conservation. Natural History Publications, Sabah, pp. 69-89.
Cogniaux, A. 1898-1902. Orchidaceae. In: C.F.P. Martius, A.G. Eichler \& I. Urban (eds.). Flora Brasiliensis. R. Oldenbourg, Monachii, v.3, pp. 1-663, t. 1-119.

Dressler, R.L. 1967. The genera Amblostoma, Lanium and Stenoglossum. Brittonia 19: 237-243.

Dressler, R.L. 1984. La delimitación de géneros en el complejo Epidendrum. Orquidea (Mexico) 9: 277-298.

Dressler, R.L. 1989. Will the real Epidendrum ibaguense please stand up? American Orchid Society Bulletin 58: 796-800.

Dunsterville, G.C.K. 1979. Orchids of Venezuela Epidendrum elongatum. American Orchid Society Bulletin 48: 447-454.

Goldman D.H., van den Berg, C. \& Griffith M.P. 2004. Morphometric circumscription of species and infraspecific taxa in Calopogon R. Br. (Orchidaceae). Plant Systematics and Evolution 247: 37-60.

Hágsater, E. 1993. Epidendrum anceps or Epidendrum secundum? Orquidea (Mexico) 13: 153-158.

Henderson, A. 2005. The methods of herbarium taxonomy. Systematic Botany 30: 456-459.

Jensen, R.J. 2003. The conundrum of morphometrics. Taxon 52: 663-671.

Lindley, J. 1852-1859. Epidendrum. In: J. Lindley (ed.). Folia Orchidacea. J. Matthews, London, pp. 1-97.

Pabst, G.F.J. \& Dungs, F. 1975. Orchidaceae Brasilienses v. 1. Kurt Schmersow, Hildesheim.

Palestina, R.A. \& Sosa, V. 2002. Morphological variation in populations of Bletia purpurea (Orchidaceae) and description of the new species B. riparia. Brittonia 54: 99-111.

Pansarin, E.R. 2000. Biologia reprodutiva e morfologia floral de espécies de Orchidaceae em diferentes ambientes no Estado de São Paulo. Dissertação de Mestrado, Universidade Estadual de Campinas, Campinas.

Sastre, C. 1990a. Epidendrum bambusiformes de Guadeloupe et de Martinique 1. L'Orchidophile 93: 149-158.

Sastre, C. 1990b. Epidendrum bambusiformes de Guadeloupe et de Martinique 2. L'Orchidophile 94: 197-203.

Shepherd, G.J. 2006. Fitopac versão 1.6.4. Universidade Estadual de Campinas, Campinas.

Wilkinson, L. 2000. Systat Statistics version 10.0 for Windows. SPSS, Chicago.

Withner, C.L. \& Harding, P.A. 2004. The Cattleyas and their relatives: The debatable Epidendrums. Timber Press, Portland. 